\title{
Die Erfindung der Magensonde
}

Ein ethnomedizinischer Beitrag zur Prioritätsfrage

Von Werner H.Stöcklin

In der Geschichte der Magensonde kommt dem großen deutschen Kliniker Adolf Kußmaul (1822-1902) zweifellos ein Ehrenplatz zu - hat er doch als erster die Qualen der Pylorusobstruktion mit Hilfe einer konsequent durchgeführten Spültechnik zu lindern vermocht.

Seine Publikation «Über die Behandlung der Magenerweiterung durch eine neue Methode mittelst der Magenpumpe» (1869) ${ }^{1}$ legt beredtes Zeugnis ab von dieser Pionierleistung. Sie hat später ihrer bahnbrechenden Bedeutung wegen in Sudhoffs «Klassiker der Medizin» Aufnahme gefunden.

Kußmauls Therapievorschlag war von besonderer Aktualität, weil die Abdominalchirurgie zu jener Zeit wenig Anlaß zu optimistischen Erwartungen bieten konnte: «Ob vielleicht die kühneren Geschlechter einer fernen Zukunft es unternehmen werden, in solchen Fällen durch Gastrotomie, Anlegung einer Magenfistel und Erweiterung der Strictur mit Messer oder Sonde radicale Erfolge zu erzielen, wer möchte diese Frage heutzutage zu beantworten wagen? Muß man doch fürchten, schon durch ihre Aufstellung leisen oder lauten Spott zu ernten ... ${ }^{2}$ Noch war offenbar nicht zu erahnen, daß schon 1879 Péan und 1881 Billroth ihr Operationsprogramm durch Magenresektionen bereichern sollten ${ }^{3}$.

Ohne Vorbehalt wird in Pschyrembels «Klinischem Wörterbuch» ${ }^{4}$ der Name Kußmaul mit dem Begriff des Magenschlauchs assoziiert. Kußmaul selber hat allerdings nie behauptet, dieses Instrument erfunden zu haben. Wenn auch nur im Kleindruck und als Fußnote, so hält er doch in seiner Mitteilung von 1869 ausdrücklich fest, daß das Modell zu der von ihm verwendeten Pumpe amerikanischen Ursprungs sei ${ }^{5}$. Und auch die amerikanische Vorrichtung geht auf eine ganze Ahnenreihe früherer Erfinder zurück.

Die Geschichte der Magensonde ist vor allem durch W. O. Leube (1879) ${ }^{6}$, W. Fleiner $(1905)^{7}$ und durch A.Korsch $(1971)^{8}$ eingehend dargestellt worden.

Während Korsch sich in seiner Dissertation bewußt darauf beschränkt, die Spuren dieses gastroenterologischen Requisits bis ins Mittelalter zurück- 
zuverfolgen, holt Leube etwas weiter aus, indem er auch die Griechen und Römer in seine Studie einbezieht. Als Ergänzung zu Leubes Schritt in die Frühzeit möchte ich hier den Nachweis erbringen, daß sich die Magensonde sogar im neolithischen Kulturgut aufzeigen läßt.

Nach Leube ${ }^{9}$ wurde die «mechanische Behandlung» der Magenkrankheiten bereits von den alten Griechen ausgeführt, die das Erbrechen gerne in den allgemeinen Therapieplan einbezogen.

Eigentliche Vorläufer der Magensonde kamen jedoch erst in der römischen Kaiserperiode zur Anwendung, wo das forcierte Erbrechen zu jedem rechten Gelage gehörte. Der Brechreiz wurde ausgelöst durch die penna (Brechfeder), das digitale vomitorium (einen ausgestopften verlängerten Handschuhfinger) und das lorum vomitorium (einen Lederriemen, der wahrscheinlich mit ekelerregenden Gerbstoffen bestrichen war).

Der arabische Arzt Abimeron Avenzoar, der 1162 im Alter von 92 Jahren in Sevilla starb, beschrieb als erster eine hohle Sonde, die bei Schluckstörungen in die Speiseröhre eingeschoben werden konnte. Nach seiner Schilderung handelte es sich dabei um eine Art Trichter aus Silber oder Zinn, der keinesfalls bis in den Magen gestoßen wurde ${ }^{10}$.

Die nächste schriftliche Angabe über die Kenntnis einer Ösophagussonde findet sich in den Opera Omnia des Hieronymus Capivaccio (1603) ${ }^{11}$. Er vervollständigt die in die Speiseröhre eingeführte Sonde durch eine Tierblase, die mit einer nährenden Flüssigkeit gefüllt ist. Diese Nährlösung kann nun mit einem gewissen Druck eingepumpt werden.

Johann Jakob Wepfer (1620-1695) nennt eine ähnliche Methode zur Behandlung von Schierlingsvergiftungen (Schlingkrämpfe!). Er empfiehlt eine «Spritze, an der ein gebogenes Rohr befestigt ist, das man über die Epiglottis hinaus hinunterstoßen kann», um warmes öl mit Wasser, sogenanntes Hydroleum, dem Patienten einzuverleiben ${ }^{12}$.

Herman Boerhaave (1668-1738) erwähnt ebenfalls im Zusammenhang mit der Therapie der Schierlingsvergiftung eine «metallische, biegsame Sonde, die über die Zunge bis zu der Vorderseite der die Wirbel bedeckenden Membran eingeführt und von dort in den Magen hinabgestoßen wird». Nach seinen Angaben dient diese Sonde auch der Instillation von Arzneien ${ }^{13}$.

Alexander Monro II (1733-1817) aus Edinburgh - dessen Vater bei Boerhaave studiert und wohl auch durch diesen von der Magensonde gehört hatte - benützte 1793 eine Magensonde mit Spritze, um einen an Speiseröhrenstriktur leidenden Mann namens Callander am Leben zu erhalten ${ }^{14}$. Laut Korsch handelte es sich hier zum erstenmal in der Geschichte der Magen- 
sonde um eine eindeutig nachweisbare Anwendung beim Menschen ${ }^{15}$. Diese Methode wurde in Edinburgh auch später häufig geübt. Alexander Monro III, der darüber berichtet, zählt eine Reihe von Indikationen auf: Ösophagusstriktur, Kardiospasmus, Lähmung der Schlundmuskulatur, Vergiftung sowie Magenblähungen infolge von Verdauungsstörungen. Der Einsatzbereich dieses Utensils wurde durch John Hunter (1728-1793), den berühmten Londoner Chirurgen, erweitert, indem er sie für das Einflößen von Arzneimitteln bei Ertrunkenen benutzte ${ }^{16}$.

Es bleibt zu erwähnen, daß die Magensonde zu jener Zeit aus einer Silberdrahtspirale bestand, die mit Leder oder Aalhaut überzogen war. An ihrer Spitze wurde gelegentlich ein Schwämmchen festgebunden zur Schonung der Ösophagusschleimhaut - eine Vorsichtsmaßnahme, die schon um 1600 von Fabricius Hildanus (1560-1634) angegeben wurde ${ }^{17}$.

In Frankreich soll sich die Verwendung der Magensonde unabhängig von der schottischen Schule durchgesetzt haben.

Wir finden bei Dominique-Jean Larrey $(1766-1842)^{18}$, dem Kriegschirurgen Napoleons I., eine «Sonde de gomme elastique œsophagienne» beschrieben, die weit in die Speiseröhre eingeführt werden konnte und als Hilfsmittel für die Ernährung bei verletzungsbedingten Schluckstörungen gebraucht wurde. Diese Sonde konnte auch durch die Nase appliziert werden und gehörte bereits 1798 auf dem ägyptischen Feldzug zum militärchirurgischen Instrumentarium.

Hinweise auf eine Magensonde, die, durch Mund und Nase eingeführt, bei Vergiftungen nützlich sein konnte, gibt auch die Dissertation eines gewissen Renault im Jahre $1803^{19}$. Viel bekannter als Renaults Vorschläge ist jedoch eine Methode der Magenspülung geworden, die Guillaume Dupuytren (1773-1835) angeregt hat. Über das Prozedere hat er selber allerdings nichts veröffentlicht, sondern lediglich in Vorlesungen seine Studenten informiert. Korsch hält es für möglich, daß Dupuytren durch Renault oder durch Arbeiten von Boerhaave auf diese Sonde aufmerksam geworden ist ${ }^{20}$.

In den USA gilt Philip Syng Physick (1768-1837), der «Vater der amerikanischen Chirurgie», als der Erfinder der Magenspülung. Er wandte sie seit 1802 bei Opiumvergiftungen an. Die Sonde hatte er während des Studiums bei John Hunter in London und bei Monro II in Edinburgh kennengelernt. Die Idee der Magenspülung soll aber spontan in ihm aufgestiegen $\operatorname{sein}^{21}$.

Um die Prioritätsansprüche bei der Erfindung einer eigentlichen Magenpumpe stritten sich 1823-25 zwei englische Chirurgen namens Francis Bush 
und Edward Jukes. Beide empfahlen ihr Instrument speziell für die Therapie der Opiumvergiftung. Der Prioritätsstreit wurde im Lancet ausgetragen und verhalf immerhin der Magensonde zu größerer Popularität, als ihr bisher zugekommen war ${ }^{22}$.

Während Bush und Jukes als Saugvorrichtung eine Spritze bzw. eine elastische Flasche vorschlugen, wurde in der Folge sowohl die Pumpe als auch die Sonde von allen möglichen Erfindern verbessert. Doch erst Adolf Kußmaul erkannte ihre Nützlichkeit bei der Behandlung chronischer Magenleiden.

Korsch hat den Versuch unternommen, die Geschichte der Magensonde stammbaumartig darzustellen ${ }^{23}$. Aus seiner Übersicht, die zahlreichen Kriterien gerecht wird, geht eindrücklich hervor, wie sehr die Entwicklung dieses scheinbar so einfachen Instrumentes mit den Namen berühmter Ärzte verknüpft ist.

Um so erstaunlicher mutet es an, daß die Erfindung einer Magensonde oder gar einer Urform des Magenschlauches ganz unabhängig von diesen großen Geistern schon Menschen steinzeitlicher Prägung gelungen ist.

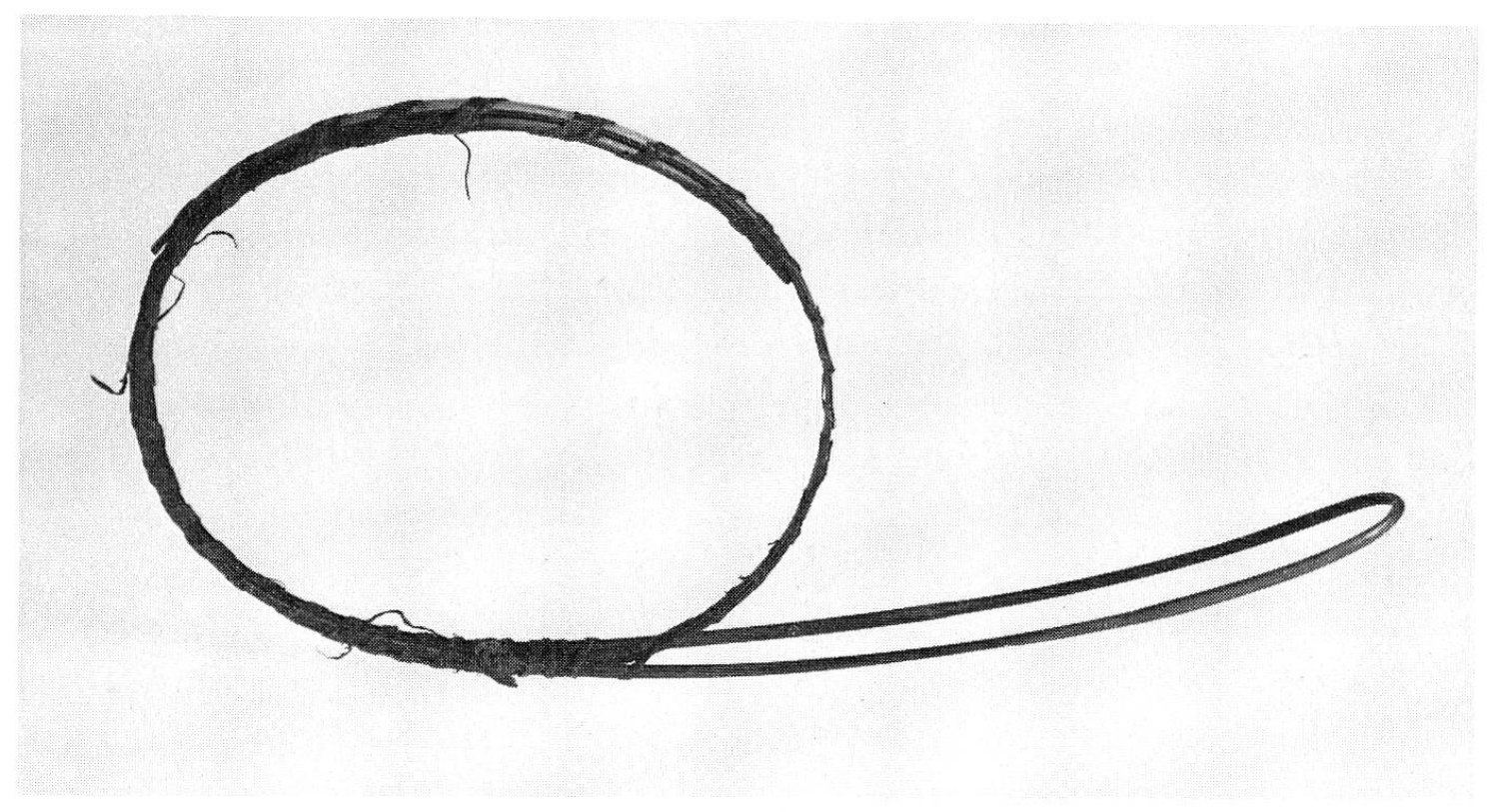

Abb.1. Eine Magenrute der Bena-Bena (östliches Hochland von Neuguinea)

Foto: Völkerkundemuseum Basel, Objekt VI 18438 
Ein solches prähistorisch-gastroenterologisches Werkzeug ist im östlichen Hochland Neuguineas zu finden. In diesem Gebiet konnte ich 1962 bei einem Stamm der Bergpapua ein von jungen Männern als Halsschmuck getragenes Gebilde erwerben, das nun unter der Bezeichnung «Magenrute» im Archiv des Basler Museums für Völkerkunde aufbewahrt wird (Nr. VI 18438). Es handelt sich dabei um eine mit Rindenbast zusammengeschnürte, U-förmig gebogene, feingeschliffene, dünne Liane, die von ihren Trägern in den Magen gestoßen werden konnte und bei verschiedenen Gelegenheiten zum Erzeugen von Erbrechen benutzt wurde (Abb. 1).

Meine eigenen Beobachtungen über den Gebrauch der Magenrute beschränken sich auf die Bena-Bena (Abb. 2), eine Sprachgruppe im Markham Valley, die zu Beginn der 1930er Jahre erstmals mit Europäern in Kontakt gekommen ist. Nach Literaturangaben ${ }^{24}$ war die Verwendung eines ähnlichen Utensils aber auch gewissen Nachbarvölkern der Bena-Bena bekannt (Gahuku-Gama-Gruppe).

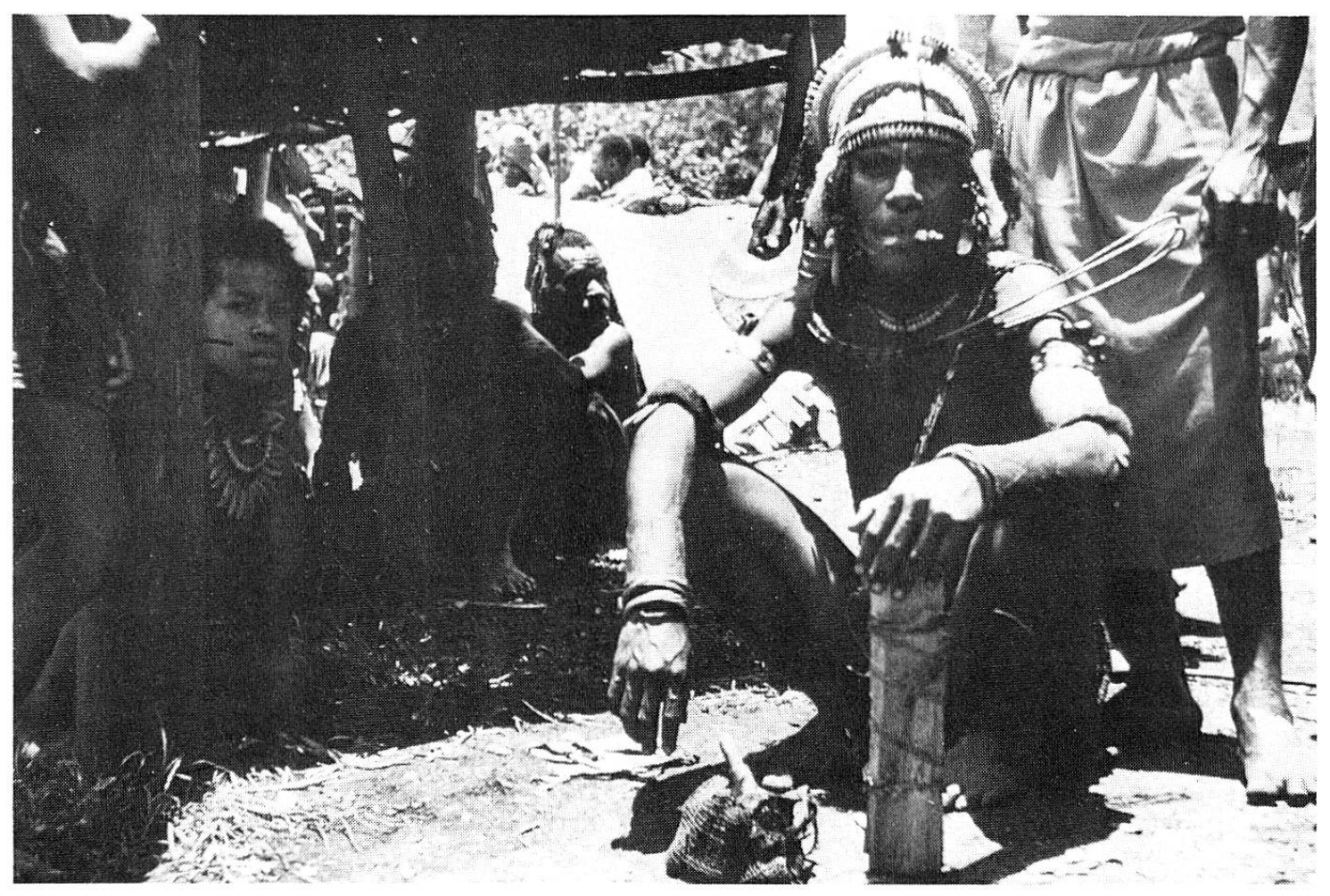

Abb.2. Junger Bena-Mann mit zwei Magenruten, die als Halsschmuck getragen werden (1962) Foto: W.Stöcklin 
In erster Linie wurde damit bei den Initiationsfeierlichkeiten eine rituelle Reinigung der Novizen bewirkt ${ }^{25}$. Daneben konnte man sich angeblich auf der Jagd nach Wildschweinen und Kasuaren den überfüllten Magen entlasten und so die Ausdauer beim Verfolgungslauf verbessern. Und schließlich war - nach den Informationen von Ronald Berndt - die Liste der Anwendungsmöglichkeiten noch durch folgende Ratschläge zu ergänzen: «Wenn eine Frau dir Nahrung gibt, nimm diese Rute, verschlucke sie und erbreche.

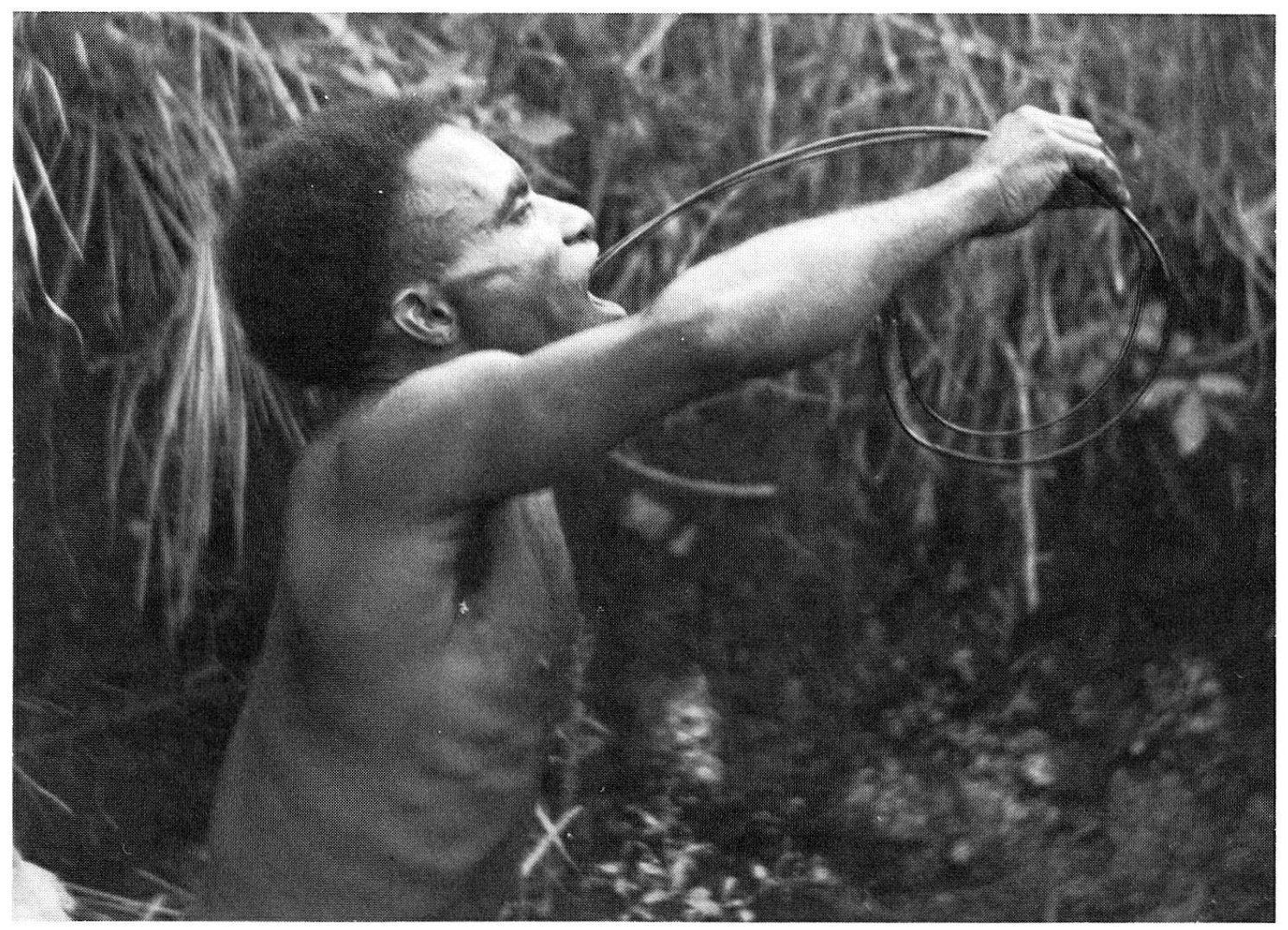

Abb.3. Typische Körperhaltung beim Einführen der Magenrute (Bena-Bena) Foto: W.Stöcklin

Wenn dir eine menstruierende Frau Speise gibt, nimm die Rute, verschlucke sie und erbreche. Wenn eine schwangere Frau dir Nahrung gibt, nimm... [usw.] Wenn ein Mann mit deiner Frau Ehebruch begeht, nimm die Rute... Denn wenn du Speisen ißt, die eine menstruierende Frau zubereitet hat, werden die Speisen in deinem Körper zu Blut. Wenn du Nahrung von einer Schwangeren annimmst, wird dein Körper anschwellen.... ${ }^{26}$ 


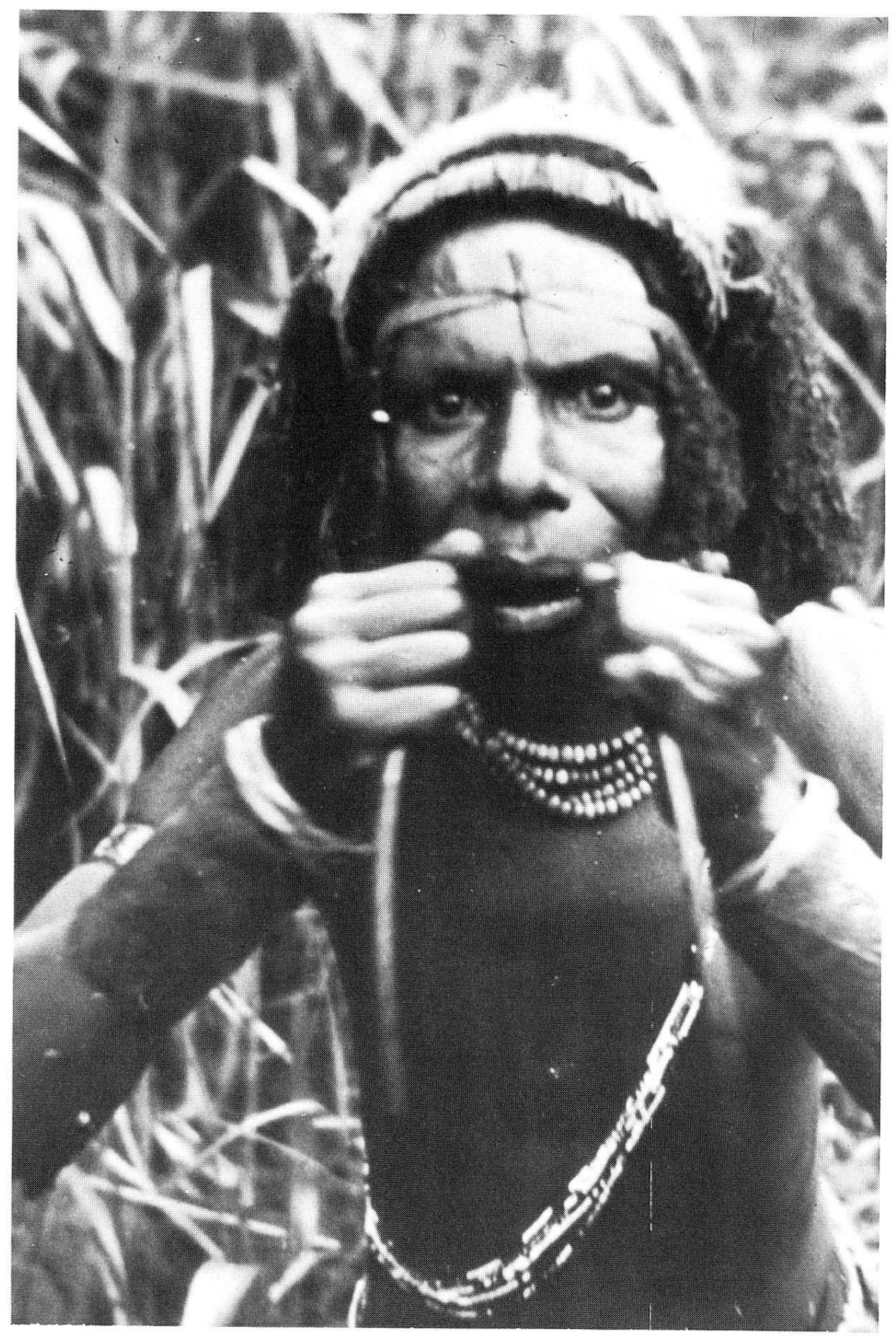

Abb.4. Nach Applikation der Magenrute wird durch rhythmische Bewegungen Brechreiz erzeugt (Bena-Bena). Foto: W. Stöcklin 
Über die Technik des Einführens dieser «Magensonde» geben Abb. 3 und 4 besser Auskunft als eine verbale Schilderung. Zumindest bei meinen Gewährsmännern hatte ein Schluck Wasser als Gleitmittel zu dienen. Die Schlinge wurde von ihrer Basthülle befreit, wie eine Wünschelrute gefaßt und mit viel Gewürge und sanfter Gewalt offensichtlich bis in den Magen hinuntergeschoben. Wie qualvoll die Prozedur für einen Ungeübten war, verriet vor allem die Mimik eines Spitalangestellten, der sich von den Sitten der Väter bereits etwas distanziert hatte. Aber auch für traditionsgebundenere Bena-Burschen war die Applikation der Magenrute kein reines Vergnügen $^{27}$.

Es ließe sich vielleicht einwenden, daß dieses «steinzeitliche» Instrument kaum mit einer Magensonde oder gar einem Magenschlauch der westlichen Medizin vergleichbar sei. Doch die genauere Inspektion der federnden Doppelsonde zeigt, daß diese unorthodoxe Magenrute das «Schlauchprinzip» noch raffinierter löst als unsere eigenen Erfindungen - wird doch durch die spreizende Spannung dieser haarnadelförmigen Liane der Ösophagus bis hinunter zur Kardia zu einem flachen, breiten Rohr gedehnt.

Von medizinischen Indikationen der Magenrute im Sinne der abendländischen Heilkunde war nichts Sicheres in Erfahrung zu bringen, obwohl die Vorstellung einer «Entgiftung» deutlich mitzuklingen schien. Dennoch wäre es denkbar, daß sich diese urtümliche Erfindung unter gegebenen Umständen zu einem eigentlichen medizinischen Hilfsmittel hätte weiterentwickeln lassen.

So sollten wir in der Geschichte der Magensonde nicht nur auf die Galerie unserer berühmten Ärzte zurückblicken, sondern bescheiden akzeptieren, daß ein zündender Funke zu unserer gastroenterologischen Technik schon in neolithischen Köpfen zustande kam. 


\section{Anmerkungen}

${ }^{1}$ Kußmaul, Adolf, Über die Behandlung der Magenerweiterung durch eine neue Methode mittelst der Magenpumpe. In: Deutsches Archiv f. klin. Med.6, 1869.

2 Kußmaul, Adolf, a. a. O., S. 485.

3 Thorwald, Jürgen, Das Jahrhundert der Chirurgen, Stuttgart 1956, S. $361 \mathrm{f}$.

4 Pschyrembel, Willibald, Klinisches Wörterbuch, Berlin 1955, S.514.

5 Kußmaul, Adolf, a. a.0., S.456.

${ }^{6}$ Leube, Wilhelm Olivier, Die Magensonde, Erlangen 1879.

${ }^{7}$ Fleiner, Wilhelm, Geschichte und Klinik der Magenspülungen. In: Deutsche Klinik am Eingang des 20. Jahrhunderts, Bd.5, Berlin und Wien 1905.

${ }^{8}$ Korsch, Albrecht, Die Geschichte der Magensonde, Diss. Köln 1971.

9 Vgl.Eulenburg, Albert, Medicinisch-chirurgisches Handwörterbuch für praktische Ärzte, Wien und Leipzig 1887, S.465.

10 Avenzoar, Abimeron, Liber Theizir. Angehängt an Colliget Averrois Cordubensis, Venedig 1497, Lib.I, Tract. X, Cap.XVIII, De privatione sensus et motus et relaxatione meri et isofagi.

11 Capivaccio, Girolamo: Hieronymi Capivaccei Pativani Opera omnia, quinque sectionibus comprehensa, Frankfurt 1603, Liber I, Cap.54.

12 Wepfer, Johann Jakob, Cicutae aquaticae historia et noxae, commentario illustrata, Basileae MDCL XXIX [Basel 1679], S. 325, 326, 252.

13 Boerhaave, Herman, Praelectiones academicae in proprias institutiones rei medicae, Bd.4, Gottingae 1744, S.388.

14 Monro III, Alexander, De dysphagia, Diss. Edinburgi 1797.

Vgl. auch: Wright-St Clair, Rex E., Doctors Monro. A medical saga. The Wellcome Historical Medical Library, London 1964, S. 17, 20, 38.

15 Korsch, A., a.a. O., S. 23.

${ }^{16}$ Hunter, John, Proposals for the recovery of people apparently drowned. Referat 1776 vor der Royal Society of Sciences, abgedruckt bei W.O.Leube, Die Magensonde, Erlangen 1879, S. 20.

17 Korsch, A., a.a. O., S. 32 .

${ }^{18}$ Larrey, Dominique-Jean, Relation historique et chirurgicale de l'expédition de l'armée d'orient en Égypte et en Syrie, Paris 1803, S.280.

19 Renault, Casimir, Nouvelles expériences sur les contre-poisons de l'arsenic, Diss. Paris 1801.

${ }^{20}$ Cadet, Félix, Secours à administrer dans les empoisonnements d'après la méthode de M. Dupuytren. Bull. de Pharm. (Paris 1810), S. 62.

${ }^{21}$ Physick, Philip Syng, Account of a new mode of extracting poisonous substances from the stomach. Eclectic Repertory, 1813, 3, S.111-113.

22 Vgl. Korsch, A., a.a. O., S. 44-48.

${ }^{23}$ Korsch, A., a.a.O., S. 52.

${ }^{24}$ Berndt, Ronald M., Excess and Restraint, Chicago 1962, S.56, Anm. 18; S.97-100.

Berndt, Ronald M., The Kamano, Usurufa, Jate and Fore of the Eastern Highlands. In: Lawrence, P., Meggitt, H. M. J. (eds.): Gods, ghosts, and men in Melanesia, Melbourne 1965, S.82.

Read, K.E., Nama cult of the Central Highlands, New Guinea. Oceania 23 (1952), No. 1, S. 13. 
${ }^{25}$ Zur Deutung dieses Brauches vgl.: Hauser-Schäublin, Brigitta, Vom Terror und Segen des Blutes. Oder: Die Emanzipation des Mannes von der Frau. In: Wiener völkerkundliche Mitteilungen, Wien 1977/78.

${ }^{26}$ Vgl. Berndt, R.M., 1962, S. 97-100. Zit. nach Hauser-Schäublin, Brigitta, 1977/78, S. 106.

${ }_{27}$ Über das Erlernen der Applikationstechnik vgl. Read, K.E., 1952, S.13: "The boys are compelled to watch and assist the older men as they demonstrate the rites of vomiting and blood-letting. They are forced to hold the ends of the u-shaped lengths of cane which their seniors have thrust down their own throats, and to move these up and down while the latter retch in the water. Later, when their own turn comes, the men exhort them with the words: 'You have done this to us; now we do it to you'..."

\section{Summary}

Kussmaul's stomach pump of 1869 had precursors. The Romans used a feather, an elongated glove-finger or a leather strap to excite vomiting. Wepfer and Boerhaave used a probe for the treatment of hemlock-poisonings. The author shows that a stomach-rod is already in use in a stone-age people. He has found this instrument among the Bena-Bena in the eastern upland of New Guinea. The illustrations show its form and its application.

Dr. med. Werner H.Stöcklin

Rößligasse 32

CH-4125 Riehen 